Pathologe 2020 - 41 (Suppl 2):S177 https://doi.org/10.1007/s00292-020-00843-3 Online publiziert: 28 . Oktober 2020 (c) Springer Medizin Verlag GmbH, ein Teil von Springer Nature 2020

Nachdem in diesem Jahr die Jahrestagung der Deutschen Gesellschaft für Pathologie (DGP) als Onlinekongress stattfand, wurde die Sitzung der Arbeitsgemeinschaft Kinder- und Fetalpathologie in diesem Rahmen als On-demand-Sitzung abgehalten. Die Poster wurden als e-Poster präsentiert. Insgesamt waren $24 \mathrm{Bei}$ träge angemeldet worden, davon wurden 16 als Vorträge angenommen und 8 als Poster.

Die Vortragssitzung wurde traditionell eröffnet mit dem Keynotevortrag. Er wurde in diesem Jahr von Klaus Busam, Memorial Sloan Kettering Cancer Center, New York, gehalten, einem internationalen Experten für pädiatrische Dermatopathologie. In seinem spannenden Übersichtsvortrag fokussierte er sich auf die diagnostischen Probleme bei der Abgrenzung melanozytärer Tumore, so z. B. dem Unterschied zwischen einem proliferativen melanozytären Knoten und einem Melanom, auch unter Berücksichtigung des kongenitalen Riesenzellnävus, oder den differenzialdiagnostischen Kriterien des gutartigen Spitz-Nävus gegenüber einem Spitz-Melanom, jeweils unter Berücksichtigung der immunhistochemischen, zytogenetischen und molekularpathologischen Methoden.

Das breite Expressionsspektrum der Cancer Testis Antigene im fetalen Gewebe und deren biologische Funktion stellten Jungbluth et al., New York, vor. Die hohe Spezifität von BCL11B und GLG1 für die Diagnose des Ewing-Sarkoms demonstrierten Orth et al., München. Über das Mikroenvironment von Immunzellen und die Expression von Stammzellmarkern in Wilms-Tumoren

Annette M. Müller

Praxis für Pathologie/Zentrum für Kinderpathologie, Uniklinik Köln, Köln, Deutschland

\title{
Bericht über die Beiträge der Arbeitsgemeinschaft Kinder- und Fetalpathologie im Rahmen der 104. Jahrestagung der DGP 2020
}

sprachen Stahl et al., Bonn. Mittels selektiver BH3-Mimetika wiesen Bierbrauer et al., Frankfurt, Heidelberg, nach, dass BCL2 und BCL-XL sowie MCL relevante therapeutische Targets im Neuroblastom sind. Miller, Augsburg, sprach über ein DICER1-mutiertes intrakranielles Sarkom und Gretser et al., Frankfurt, über ein CHEK1- und NF1-mutiertes malignes peritoneales Mesotheliom. Tierexperimentelle Untersuchungen zur Prävention der intraoperativen Myokardischämie stellten Preuße et al., Bonn, Köln, vor. Das breite Spektrum des fetalen Phänotyps und die genetische Heterogenität des primären „isolierten“ und „syndromalen" Mikrocephalus demonstrierten Schoner et al., Marburg, Wien, anhand einer Fallserie. Eine Studie zur Bedeutung von CD56, CD16, CD4 und CD8 im Endometrium und peripheren Blut bei Implantationsversagen im Rahmen der assistierten Reproduktion stellten Müller et al., Köln, Bonn, vor. Die Expression des Komplementrezeptors CD11C/CD18 bei der chronischen histiozytären Intervillositis präsentierten Müller et al., Köln, Hannover. Gradhand, Frankfurt, sprach über Unterschiede in der pädiatrischen und perinatalen Pathologie in Großbritannien und Deutschland. Pavlov et al., Köln, demonstrierten die Korrelation zwischen Nabelschnurlänge und $\mathrm{Na}$ belschnurcoiling bei Gestationsdiabetes. Reinehr et al., Zürich, präsentierten ein bisher nur in wenigen Berichten publiziertes Van-Meldergem-Syndrom. Saleh et al., Köln, sprachen über die Definition des Placenta-accreta-Spektrums in Abgrenzung zur „scar pregnancy“. Brada et al., Zürich, präsentierten eine nach ihrer Literatursuche bisher nicht beschriebene komplexe Darmrotation.

Themen der als $e$-Poster präsentierten Poster waren die Non-compaction-Kardiomyopathie (Hager et al., Essen), die differenzialdiagnostischen Probleme bei der Diagnostik der interstitiellen Lungenfibrose nach Chemotherapie (Müller et al., Köln) sowie bei multiplen Fehlbildungen bei der Diagnostik des FrynsSyndroms (Erbel et al., Freiburg), die seltene Manifestation einer nichtparasitären Milzzyste bei Kindern (Müller und Heydweiller, Köln, Bonn), der Stellenwert einer kritischen Laryngotrachealstenose beim Fraser-Syndrom (Fischer et al., Gera), die Diagnostik des Ischiopagus in der 15. Schwangerschaftswoche (Eich et al., Köln) und ein konstitutionelles Mismatch-repair-deficiency-Syndrom mit bialleler Mutation im PMS2-Gen bei multiplen Neoplasien (Walter et al., Frankfurt).

\section{Korrespondenzadresse}

Prof. Dr. med. Annette M. Müller

Praxis für Pathologie/Zentrum für Kinderpathologie, Uniklinik Köln Kerpener Str. 62, 50937 Köln, Deutschland annette.mueller@uk-koeln.de

\section{Einhaltung ethischer Richtlinien}

Interessenkonflikt. A. M. Müller gibt an, dass kein Interessenkonflikt besteht.

Für die aufgeführten Studien lag jeweils ein positives Votum der jeweils zuständigen Ethikkommission vor.

The supplement containing this article is not sponsored by industry. 